\title{
Area methods, extremal problems and extremal domains for pairs of conformal mappings
}

\author{
by KaZimierz Wlodarczyk (Lódź)
}

\begin{abstract}
In the classes of pairs $, F, G$, of functions $F$ and $G$ analytic and univalent in the unit disc and satisfying the condition $F(z) G(\zeta) \neq 1$ there (generating, in the special case. bounded, Bieberbach-Eilenberg and Grunsky - Shah functions) or the condition $F(z)+G(\zeta) \neq 0$ (generating, in the special case, Gel'fer functions), by applying coefficient inequalities of Grunsky Nehari type, a characterization of extremal pairs maximizing functionals dependent on the derivatives $F^{(n)}$ and $G^{(n)}, n=2,3$, is given.
\end{abstract}

1. Introduction, notations and statement of the results. The present paper deals with the class $C$ of pairs $\{F, G\}$ of functions

$$
F(z)=a+a_{1}+a_{2} z^{2}+\ldots, \quad G(z)=b+b_{1} z+b_{2} z^{2}+\ldots
$$

univalent in $\Delta=\{z:|z|<1\}$ and satisfying the condition

$$
F(z) G(\zeta) \neq 1
$$

for all $z, \zeta$ belonging to 4 , and the class $D$ of pairs $\{F, G\}$ of functions

$$
F(z)=1+2 a_{1} z+2 a_{2} z^{2}+\ldots, \quad G(z)=1+2 b_{1} z+2 b_{2} z^{2}+\ldots
$$

univalent in $\Delta$ and satisfying the condition

$$
F(z)+G(\zeta) \neq 0
$$

for all $z, \zeta$ belonging to $\Delta$. These classes, some other classes to them as well as some their subclasses have been intensely investigated since 1934 (cf. e.g., $[1]-[3]$ and [5]-[12]).

Let $\hat{K}, K, L_{\zeta}, P$ and $Q$ stand for mappings defined as follows:

$$
\begin{gathered}
\hat{K}(z)=\left[z /(z-1)^{2}\right][1+a b-\sqrt{a b}(z+1 / z)], \quad K(z)=z /(z-1)^{2}, \\
L_{\zeta}(z)=z /[(z-\zeta)(z+\bar{\zeta})] . \quad P(z)=(z-1) /(z+1), \quad Q(z)=1 / z-z,
\end{gathered}
$$

and let

$$
A_{n}=a_{n} / a_{1}, \quad B_{n}=b_{n} / b_{1}, \quad n=2,3 .
$$


If $z_{1}, z_{2}$ and $\{F, G\}$ belong to $\Delta$ and $C$, respectively, let $\lambda\left(a, b, z_{1}, z_{2}, F, G\right)$

$$
\begin{gathered}
=\left[\frac{F^{\prime \prime}\left(z_{1}\right)}{F^{\prime}\left(z_{1}\right)}+2 \frac{G\left(z_{2}\right)+2 \sqrt{b / a}}{1-F\left(z_{1}\right) G\left(z_{2}\right)} F^{\prime}\left(z_{1}\right)\right]\left(1-\left|z_{1}\right|^{2}\right)-2 \bar{z}_{1}, \quad a, b \neq 0, \\
=\left[\frac{F^{\prime \prime}\left(z_{1}\right)}{F^{\prime}\left(z_{1}\right)}+2 \frac{G\left(z_{2}\right)+2}{1-F\left(z_{1}\right) G\left(z_{2}\right)} F^{\prime}\left(z_{1}\right)\right]\left(1-\left|z_{1}\right|^{2}\right)-2 \bar{z}_{1}, \quad a=b=0, \\
v\left(a, b, z_{1}, z_{2}, F, G\right)=\frac{1-F\left(z_{1}\right) G\left(z_{2}\right)}{b(1-a b) F^{\prime}\left(z_{1}\right)\left(1-\left|z_{1}\right|^{2}\right)}, \quad a, b \neq 0, \\
=\frac{1-F\left(z_{1}\right) G\left(z_{2}\right)}{F^{\prime}\left(z_{1}\right)\left(1-\left|z_{1}\right|^{2}\right)}, \quad a=b=0,
\end{gathered}
$$

and, if $z_{1}, z_{2}$ and $\{F, G\}$ belong to $\Delta$ and $D$, respectively, let

where

$$
\begin{aligned}
& \varphi\left(z_{1}, z_{2}, F, G\right)=\left[\frac{F^{\prime \prime}\left(z_{1}\right)}{F^{\prime}\left(z_{1}\right)}+4 \frac{F^{\prime}\left(z_{1}\right)}{F\left(z_{1}\right)+G\left(z_{2}\right)}\right]\left(1-\left|z_{1}\right|^{2}\right)-2 \bar{z}_{1}, \\
& \chi\left(z_{1}, z_{2}, F, G\right)=\left[\left\{F ; z_{1}\right\}+24 \frac{F^{\prime 2}\left(z_{1}\right)}{\left[F\left(z_{1}\right)+G\left(z_{2}\right)\right]^{2}}\right]\left(1-\left|z_{1}\right|^{2}\right)^{2}, \\
& \psi\left(z_{1}, z_{2}, F, G\right)=\frac{F\left(z_{1}\right)+G\left(z_{2}\right)}{F^{\prime}\left(z_{1}\right)\left(1-z_{1}^{2}\right)},
\end{aligned}
$$

$$
\left\{F ; z_{1}\right\}=\frac{F^{\prime \prime \prime}\left(z_{1}\right)}{F^{\prime}\left(z_{1}\right)}-\frac{3}{2}\left(\frac{F^{\prime \prime}\left(z_{1}\right)}{F^{\prime}\left(z_{1}\right)}\right)^{2} .
$$

In this paper we investigate a problem of finding the sharp estimate of the functionals

$$
\begin{aligned}
\lambda=\left|\lambda\left(a, b, z_{1}, z_{2}, F, G\right) v\left(a, b, z_{1}, z_{2}, F, G\right)\right|+ \\
+\left|\lambda\left(b, a, z_{2}, z_{1}, G, F\right) v\left(b, a, z_{2}, z_{1}, G, F\right)\right|,
\end{aligned}
$$

where $\{F, G\}$ ranges over $C$, and

$$
\begin{aligned}
& \varphi=\left|\varphi\left(z_{1}, z_{2}, F, G\right) \psi\left(z_{1}, z_{2}, F, G\right)\right|+\left|\varphi\left(z_{2}, z_{1}, G, F\right) \psi\left(z_{2}, z_{1}, G, F\right)\right|, \\
& \chi=\left|\chi\left(z_{1}, z_{2}, F, G\right) \psi^{2}\left(z_{1}, z_{2}, F, G\right)\right|+\left|\chi\left(z_{2}, z_{1}, G, F\right) \psi^{2}\left(z_{2}, z_{1}, G, F\right)\right|,
\end{aligned}
$$

where $\{F, G\}$ ranges over $D$.

For $\left\{F^{*}, G^{*}\right\}$ belonging to $C$, we define

$$
\begin{aligned}
F^{* *}(z) & =\frac{a[1-f(0) g(0)]-(1-a b) f(0)+[1-a b f(0) g(0)] f(z)}{1-a b f(0) g(0)+\{b[1-f(0) g(0)]-(1-a b) g(0)\} f(z)}, \\
f(z) & =\frac{F^{*} \circ p(z)-a}{1-b F^{*} \circ p(z)},
\end{aligned}
$$




$$
\begin{aligned}
G^{* *}(z) & =\frac{b[1-f(0) g(0)]-(1-a b) g(0)+[1-a b f(0) g(0)] g(z)}{1-a b f(0) g(0)+\{a[1-f(0) g(0)]-(1-a b) f(0)\} g(z)} \\
g(z) & =\frac{G^{*} \circ q(z)-b}{1-a G^{*} \circ q(z)}
\end{aligned}
$$

and for $\left\{F^{*}, G^{*}\right\}$ belonging to $D$, we define

$$
\begin{aligned}
F^{* *}(z) & =\frac{[1-f(0) g(0)][1-f(z)]}{1+f(0) g(0)-2 f(0)+[1+f(0) g(0)-2 g(0)] f(z)}, \\
f(z) & =\frac{F^{*} \circ p(z)-1}{F^{*} \circ p(z)+1}, \\
G^{* *}(z) & =\frac{[1-f(0) g(0)][1-g(z)]}{1+f(0) g(0)-2 g(0)+[1+f(0) g(0)-2 f(0)] g(z)}, \\
g(z) & =\frac{G^{*} \circ q(z)-1}{G^{*} \circ q(z)+1},
\end{aligned}
$$

where

$$
p(z)=\left(z-z_{1}\right) /\left(1-\bar{z}_{1} z\right), \quad q(z)=\left(z-z_{2}\right) /\left(1-\bar{z}_{2} z\right) .
$$

The results obtained in this paper will be formulated as follows.

Theorem 1. If $\{F, G\}$ belongs to $C$, then the corresponding $\lambda$ does not exceed

$$
\lambda^{*}=4\left(\left|v\left(a, b, z_{1}, z_{2}, F, G\right)\right|+\left|v\left(b, a, z_{2}, z_{1}, G, F\right)\right|\right) .
$$

The estimate is sharp for each $a$ and $b$ such that $a b \geqslant 0$. All the extremal pairs are given by formulae (1.1) and (1.1') with the functions $F^{*}, G^{*}$ satisfying the equations (Figure 1)

$$
\begin{aligned}
& F^{*}(z)=\sqrt{\frac{a}{b}} \hat{K}^{-1}\left[\frac{b a_{1}}{\sqrt{a b}} \frac{1+\sqrt{a b}}{1-\sqrt{a b}} K\left(e^{\alpha i} z\right)\right], \\
& G^{*}(z)=\sqrt{\frac{b}{a}} \hat{K}^{-1}\left[\frac{a b_{1}}{\sqrt{a b}} \frac{1+\sqrt{a b}}{1-\sqrt{a b}} K\left(e^{\beta i} z\right)\right]
\end{aligned}
$$

when $a b>0$, where $\operatorname{sgn}\left\{b a_{1}\right\}=\operatorname{sgn}\left\{a b_{1}\right\}=\operatorname{sgn}\{1-\sqrt{a b}\},-\pi<\alpha, \beta \leqslant \pi$, and the equations

$$
F^{*}(z)=K^{-1}\left[a_{1} K\left(e^{a i} z\right)\right], \quad G^{*}(z)=K^{-1}\left[b_{1} K\left(e^{\beta i} z\right)\right]
$$

when $a=b=0$, where $a_{1}>0, b_{1}>0,-\pi<\alpha, \beta \leqslant \pi$. exceed

THEOREM 2. If $\{F, G\}$ belongs to $D$, then the corresponding $\varphi$ does not

$$
\varphi^{*}=2\left(\left|\psi\left(z_{1}, z_{2}, F, G\right)\right|+\left|\psi\left(z_{2}, z_{1}, G, F\right)\right|\right)
$$


The estimate is sharp. All the extremal pairs are given by formulae (1.2), with the functions $F^{*}, G^{*}$ satisfying the equations (Figure 2)

$$
F^{*}(z)=P^{-1}\left\{K^{1}\left[a_{1} K\left(e^{a i} z\right)\right]\right\}, \quad G^{*}(z)=P^{-1}\left\{K^{-1}\left[b_{1} K\left(e^{\beta i} z\right)\right]\right\},
$$

where $a_{1}>0, b_{1}>0,-\pi<\alpha, \beta \leqslant \pi$.

TheOREM 3. If $\{F, G\}$ belongs to $D$, then the corresponding $\chi$ does not exceed

$$
\chi^{*}=6\left(\left|\psi^{2}\left(z_{1}, z_{2}, F, G\right)\right|+\left|\psi^{2}\left(z_{2}, z_{1}, G, F\right)\right|\right) .
$$

The estimate is sharp. All the extremal pairs are given by formulae (1.2), with that the functions $F^{*}, G^{*}$ satisfy the equations (Figure 3)

$$
F^{*}(z)=Q^{-1}\left[4 a_{1} L_{\zeta_{1}}\left(e^{\alpha i} z\right)\right], \quad G^{*}(z)=Q^{-1}\left[4 b_{1} L_{\zeta_{2}}\left(e^{\beta i} z\right)\right],
$$

where $a_{1}>0, b_{1}>0,-\pi<\alpha, \beta \leqslant \pi,\left|\zeta_{1}\right|=\left|\zeta_{2}\right|=1$,

$$
\begin{array}{ll}
A_{2}-a_{1}=-2 \operatorname{Im}\left\{\zeta_{1}\right\} i, & \left|\operatorname{Im}\left\{\zeta_{1}\right\}\right| \leqslant 1-a_{1}, \\
B_{2}-b_{1}=-2 \operatorname{Im}\left\{\zeta_{2}\right\} i, & \left|\operatorname{Im}\left\{\zeta_{2}\right\}\right| \leqslant 1-b_{1} .
\end{array}
$$

Thus, for every $a_{1}, b_{1}$, the functions $F^{*}, G^{*}$ belong to one-parameter families, where the parameters are $a_{2}, b_{2}$ or $\zeta_{1}, \zeta_{2}$, respectively.

The paper ends with a few applications and remarks. In particular, we obtain the sharp estimate for bounded, Bieberbach-Eilenberg, GrunskyShah and Gel'fer functions.

2. Proofs. If $g_{k}, k=1,2$, are mappings defined by the formulae

$$
\begin{array}{rlrl}
g_{1}(w) & =\frac{w}{a(1-b w)}-\frac{1}{b(w-a)}, & a, b \neq 0, \\
& =w-1 / w, & a=b & =0,
\end{array}
$$

when $\left.{ }_{i}^{\prime} F, G\right\}$ belongs to $C$, and

$$
g_{2}(w)=-\frac{1}{w-1}-\frac{1}{w+1}
$$

when $\{F, G\}$ belongs to $D$, and if

$$
\begin{aligned}
\sum_{q=-\infty}^{\infty} c_{q}^{1} z^{q} & =g_{1}[F(z)], & \sum_{q=-\infty}^{\infty} c_{q}^{2} z^{q} & =g_{1}[1 / G(z)], \\
& =g_{2}[F(z)], & & =g_{2}[-G(z)],
\end{aligned}
$$

where $0<|z|<1$, then

$$
\sum_{q=-\infty}^{\infty} q\left(\left|c_{q}^{1}\right|^{2}+\left|c_{q}^{2}\right|^{2}\right) \leqslant 0
$$


in virtue of (2.4), (2.6), (2.11) and (2.7) of paper [15]. In particular,

$$
\left|c_{1}^{1}\right|^{2}+\left|c_{1}^{2}\right|^{2} \leqslant\left|c_{-1}^{1}\right|^{2}+\left|c_{-1}^{2}\right|^{2}
$$

and the equality holds if and only if

$$
c_{q}^{1}=c_{q}^{2}=0, \quad q=2,3, \ldots
$$

and, if $-\pi<\varepsilon, \tau \leqslant \pi$, then

$$
\operatorname{Re}\left\{c_{1}^{1} c_{-1}^{1} \exp (\varepsilon i)+c_{1}^{2} c_{-1}^{2} \exp (\tau i)\right\} \leqslant\left|c_{-1}^{1}\right|^{2}+\left|c_{-1}^{2}\right|^{2}
$$

in virtue of the Cauchy-Schwarz inequality and (2.5), with the equality holding if and only if

$$
c_{1}^{1}=\bar{c}_{-1}^{1} \exp (-\varepsilon i), \quad c_{1}^{2}=\bar{c}_{-1}^{2} \exp (-\tau i) .
$$

Proof of Theorem 1. For $a b \neq 0$, from (2.1) and (2.3) we have

$$
\begin{array}{lll}
c_{1}^{1}=\alpha_{3} /\left(b a_{1}\right), & c_{-1}^{1}=-1 /\left(b a_{1}\right), & c_{0}^{1}=\alpha_{2} /\left(b a_{1}\right), \\
c_{1}^{2}=-\beta_{3} /\left(a b_{1}\right), & c_{-1}^{2}=1 /\left(a b_{1}\right), & c_{0}^{2}=-\beta_{2} /\left(a b_{1}\right),
\end{array}
$$

where

$$
\alpha_{3}=A_{3}-A_{2}^{2}+\frac{b}{a(1-a b)^{2}}, \quad a_{3}^{2}=B_{3}-B_{2}^{2}+\frac{a}{b} \frac{b_{1}^{2}}{(1-a b)^{2}} .
$$

Since $\{F, G\}$ belongs to $C$ if and only if $\{\hat{F}, \hat{G}\}$ belongs to $C$, where

$$
\begin{array}{ll}
\hat{F}(z)=\frac{\sqrt{a}+f(z)}{1+\sqrt{b} f(z)}, & f(z)=\left[\frac{F\left(z^{2}\right)-a}{1-b F\left(z^{2}\right)}\right]^{1 / 2}, \\
\hat{G}(z)=\frac{\sqrt{b}+g(z)}{1+\sqrt{a} g(z)}, & g(z)=\left[\frac{G\left(z^{2}\right)-b}{1-a G\left(z^{2}\right)}\right]^{1 / 2},
\end{array}
$$

therefore, if $\hat{F}(z)=\hat{a}+\hat{a}_{1} z+\hat{a}_{2} z^{2}+\ldots, \hat{G}(z)=\hat{b}+\hat{b}_{1} z+\hat{b}_{2} z^{2}+\ldots$, then it follows from (2.7) and (2.9) that

$$
-\operatorname{Re}\left\{e^{c i} \frac{\hat{\alpha}_{3}}{\left(\bar{b} \hat{a}_{1}\right)^{2}}+e^{r i} \frac{\hat{\beta}_{3}}{\left(\hat{a} \hat{b}_{1}\right)^{2}}\right\} \leqslant \frac{1}{\left|\hat{b} \hat{a}_{1}\right|^{2}}+\frac{1}{\left|\hat{a} \hat{b}_{1}\right|^{2}}
$$

In consequence, from this, (2.10), and because $\varepsilon, \tau$ are arbitrary we get that

$$
\begin{aligned}
\left|A_{2}+\frac{(b+2 \sqrt{b / a}) a_{1}}{1-a b}\right|\left|a b_{1}\right|+\left|B_{2}+\frac{(a+2 \sqrt{a / b}) b_{1}}{1-a b}\right| & \left|b a_{1}\right| \\
& \leqslant 2\left(\left|b a_{1}\right|+\left|a b_{1}\right|\right)
\end{aligned}
$$

Now, note that in (2.11), along with the extremal pair $\left\{\hat{F}^{*}, \hat{G}^{\star}\right\}$, also 
the pairs $\left\{\hat{F}_{\alpha}^{*}, \hat{G}_{\beta}^{*}\right\},-\pi<\alpha, \beta \leqslant \pi, \hat{F}_{\alpha}^{*}=\hat{F}^{*}\left(e^{\alpha i} z\right), \hat{G}_{\beta}^{*}(z)=\hat{G}^{*}\left(e^{\beta i} z\right)$ are extremal. So, assume that $\left\{\hat{F}^{*}, \hat{G}^{*}\right\}$ is an extremal pair for which $\hat{b} \hat{a}_{1}>0$, $\hat{a} \hat{b}_{1}>0$, and let $\varepsilon=\tau=\pi$. Then, by (2.1), (2.3), (2.6), (2.8) and (2.9),

$$
\hat{g}_{1} \circ \hat{F}^{*}(z)=\frac{1}{\hat{b} \hat{a}_{1}} \frac{z^{2}-1}{z}, \quad-\hat{g}_{1} \circ \frac{1}{\hat{G}^{*}(z)}=\frac{1}{\hat{a} \hat{b}_{1}} \frac{z^{2}-1}{z},
$$

whence, by squaring the above equalities and replacing $z^{2}$ by $z$ in them, we obtain (1.3).

Moreover, let observe that $\left\{F^{* *}, G^{* *}\right\}$ belongs to $C$ if and only if $\left\{F^{*}, G^{*}\right.$, belongs to $C$, with

$$
\begin{aligned}
& F^{*}(z)=\frac{a+f(z)}{1+b f(z)}, \quad f(z)=\frac{F^{* *} \circ p^{-1}(z)-F^{* *}\left(z_{1}\right)}{1-F^{* *} \circ p^{-1}(z) G^{* *}\left(z_{2}\right)}, \\
& G^{*}(z)=\frac{b+g(z)}{1+a g(z)},
\end{aligned}
$$

which is equivalent to (1.1). From this and (2.12) the inequality $\lambda \leqslant \lambda^{*}$ follows.

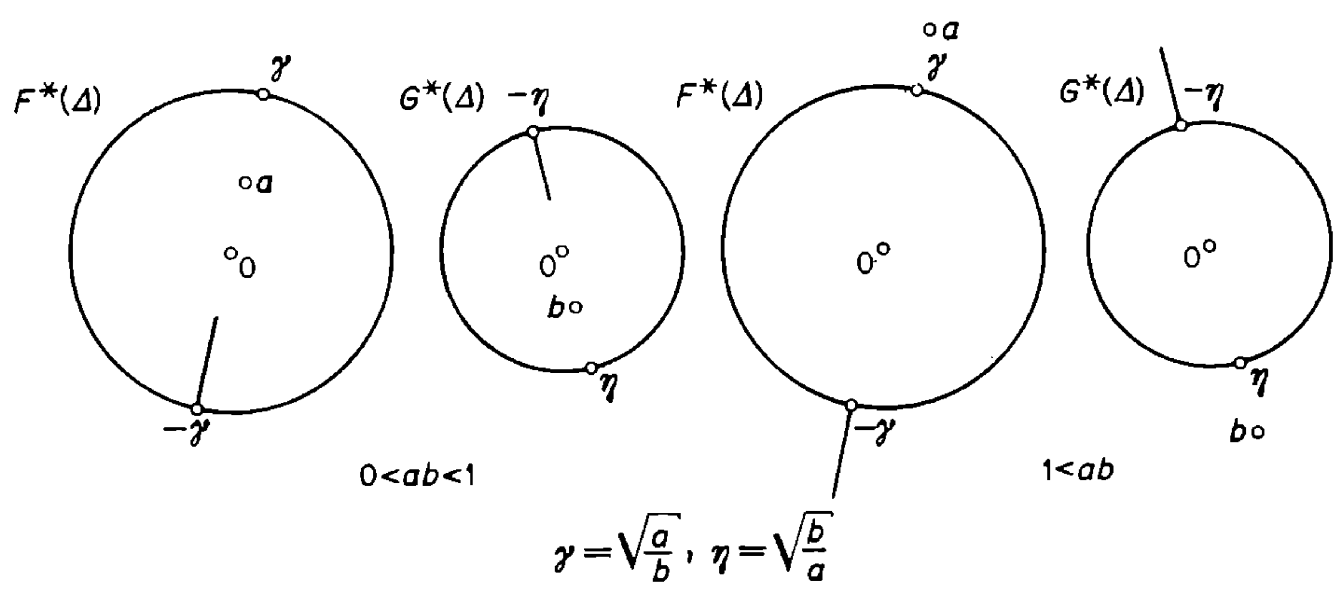

Fig. 1

The case $a=b=0$ is proved in an analogous way.

Proof of Theorem 2. From (2.2) and (2.3) we have

$$
\begin{aligned}
& c_{1}^{1}=\left(A_{3}-A_{2}^{2}+a_{1}^{2}\right) /\left(2 a_{1}\right), \quad c_{-1}^{1}=-1 /\left(2 a_{1}\right), \quad c_{0}^{1}=\left(A_{2}-a_{1}\right) /\left(2 a_{1}\right), \\
& c_{1}^{2}=-\left(B_{3}-B_{2}^{2}+b_{1}^{2}\right) /\left(2 b_{1}\right), \quad c_{-1}^{2}=1 /\left(2 b_{1}\right), \quad c_{0}^{2}=-\left(B_{2}-b_{1}\right) /\left(2 b_{1}\right),
\end{aligned}
$$

and since $\{F, G\}$ belongs to $D$ if and only if $\{\hat{F}, \hat{G}\}$ belongs to $D$, where 


$$
\begin{array}{ll}
\hat{F}(z)=\frac{1+f(z)}{1-f(z)}, & f(z)=\left[\frac{F\left(z^{2}\right)-1}{F\left(z^{2}\right)+1}\right]^{1 / 2}, \\
\hat{G}(z)=\frac{1+g(z)}{1-g(z)}, & g(z)=\left[\frac{G\left(z^{2}\right)-1}{G\left(z^{2}\right)+1}\right]^{1 / 2},
\end{array}
$$

therefore, if $\hat{F}(z)=1+2 \hat{a}_{1} z+2 \hat{a}_{2} z^{2}+\ldots, \hat{G}(z)=1+2 \hat{b}_{1} z+2 \hat{b}_{2} z^{2}+\ldots$, then from (2.7) and (2.13) it follows that

$$
-\operatorname{Re}\left\{e^{\varepsilon i} \frac{\hat{A}_{3}-\hat{A}_{2}^{2}+\hat{a}_{1}^{2}}{\hat{a}_{1}^{2}}+e^{\tau i} \frac{\hat{B}_{3}-\hat{B}_{2}^{2}+\hat{b}_{1}^{2}}{\hat{b}_{1}^{2}}\right\} \leqslant \frac{1}{\left|\hat{a}_{1}\right|^{2}}+\frac{1}{\left|\hat{b}_{1}\right|^{2}} .
$$

From this and (2.14) we obtain the inequality

$$
\left|\left(A_{2}+a_{1}\right) b_{1}\right|+\left|\left(B_{2}+b_{1}\right) a_{1}\right| \leqslant 2\left(\left|a_{1}\right|+\left|b_{1}\right|\right) .
$$

Assume that $\left\{\hat{F}^{*}, \hat{G}^{*}\right\}$ is an extremal pair in (2.15) with $\hat{a}_{1}>0, \hat{b}_{1}>0$, $\varepsilon=\tau=\pi$. Then it follows from (2.1), (2.3), (2.6), (2.8) and (2.13) that

$$
g_{2}\left[\hat{F}^{*}(z)\right]=\frac{1}{2 \sqrt{a_{1}}} \frac{1-z^{2}}{z}, \quad-g_{2}\left[-\hat{G}^{*}(z)\right]=\frac{1}{2 \sqrt{b_{1}}} \frac{1-z^{2}}{z} .
$$

By squaring the above equations and replacing $z^{2}$ by $z$ in them, we immediately obtain (1.4).

Since $\left\{F^{* *}, G^{* *}\right\}$ belongs to $D$ if and only if $\left\{F^{*}, G^{*}\right\}$ belongs to $D$, where

$$
\begin{aligned}
& F^{*}(z)=\frac{1+f(z)}{1-f(z)}, \quad f(z)=\frac{F^{* *} \circ p^{-1}(z)-F^{* *}\left(z_{1}\right)}{F^{* *} \circ p^{-1}(z)+G^{* *}\left(z_{2}\right)}, \\
& G^{*}(z)=\frac{1+g(z)}{1-g(z)}, \quad g(z)=\frac{G^{* *} \circ q^{-1}(z)-G^{* *}\left(z_{2}\right)}{G^{* *} \circ q^{-1}(z)+F^{* *}\left(z_{1}\right)},
\end{aligned}
$$

which is equivalent to (1.2), therefore, by applying (1.2) to (2.16), we obtain the inequality $\varphi \leqslant \varphi^{*}$.

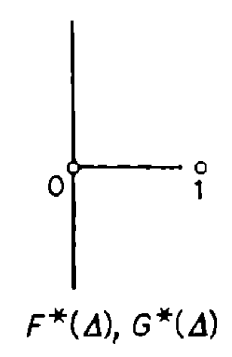

Fig. 2

Proof of Theorem 3. It follows from (2.15) that

$$
\left|\left(A_{3}-A_{2}^{2}+a_{1}^{2}\right) b_{1}^{2}\right|+\left|\left(B_{3}-B_{2}^{2}+b_{1}^{2}\right) a_{1}^{2}\right| \leqslant\left|a_{1}\right|^{2}+\left|b_{1}\right|^{2}
$$


if $\{F, G\}$ belongs to $D$; by Theorem 9 [16], the equality in (2.18) takes place only for pairs satisfying equations (1.5). The inequality $\chi \leqslant \chi^{*}$ follows from (2.18) after applying (2.17) and (1.2).
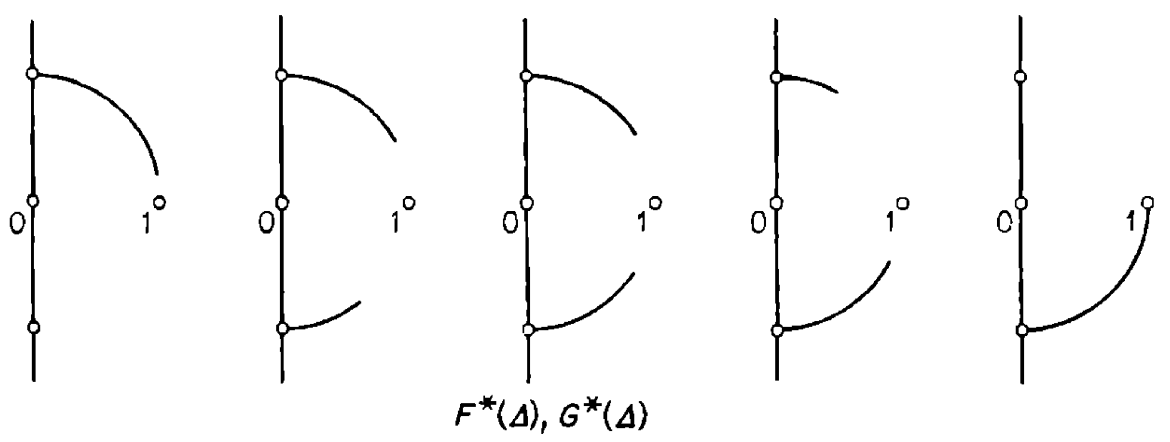

Fig. 3

3. Conclusions. Let $S_{1}$ denote the class of all functions $F(z)=b+$ $b_{1} z+\ldots+b_{n} z^{n}+\ldots$ univalent in $\Delta$ and such that $|F(z)|<1$ for $z$ belonging to $\Delta$. From Theorem 1, if $G=\bar{F}, \bar{F}(z)=\overline{F(\bar{z})}$ and $z_{1}=\bar{z}_{2}=z_{0}$, we obtain at once the sharp result and all extremal functions in the class $S_{1}$.

The function $F(z)=b_{1} z+b_{2} z^{2}+\ldots$, univalent in $\Delta$, is called a Bieberbach-Eilenberg function, respectively a Grunsky-Shah function, if $F(z) F(\zeta) \neq 1$, respectively $F(z) \overline{F(\zeta)} \neq-1$, for all $z, \zeta$ belonging to $\Delta$. From Theorem 1 , if $G=F$ and $z_{1}=z_{2}=z_{0}$, respectively, $G=-\bar{F}$ and $z_{1}=\bar{z}_{2}$ $=z_{0}$, we obtain the sharp estimate and all extremal functions in the class of Bieberbach-Eilenberg functions, and in the class of Grunsky-Shah functions, respectively.

By $S\left(b_{1}\right)$ we denote a subclass of the class $S_{1}$ of those functions $F$ for which $b=0,0<b_{1} \leqslant 1, b_{1}-$ fixed. Since, if $F$ belongs to $S\left(b_{1}\right)$, then also $F_{a}$ belongs to $S\left(b_{1}\right)$, where $F_{a}(z)=\exp (-\alpha i) F[\exp (\alpha i) z],-\pi<\alpha \leqslant \pi$, therefore, the welk-known sharp estimate in the class $S\left(B_{1}\right)$ now follows from Theorem 1 (see e.g., [13], [14]): $\left|b_{2}\right| \leqslant 2 b_{1}\left(1-b_{1}\right)$.

The function $F(z)=1+2 a_{1} z+2 a_{2} z^{2}+\ldots$, univalent in $\Delta$ is said to belong to the Gel'fer class if $F(z)+F(\zeta) \neq 0$ for $z$, $\zeta$ belonging to $\Delta$. From Theorem 2 and from the estimate $\chi \leqslant \chi^{*}$ when $G=F$ and $z_{1}=z_{2}=z_{0}$ we obtain the results of Grinšpan and Kolomojceva [4] and Gel'fer [3].

\section{Referemes}

[1] D. W. De Temple, An area method for systems of univalent functions whose ranges do not overlap, Math. Z. 128 (1972), 23-33.

[2] -, and D. B. Oult on, Formulas for the Nehari coefficients of bounded univalent functions, Canad. J. Math. 39(1977), 587-605. 
[3] S. A. Gel'fer, O klasse reguljarnych funkcij, ne prinimajuscich ni odnoj pary znađenij w i $-w$, Mat. Sb. 19(1946), 33-46 (in Russian).

[4] A. Z. Grinšpan i Z. V. Kolomojceva, Nekotorye ocenki v klasse funkcji, ne prinimajušxich ni odnoj pary znaženij wi $i$, Vestn. Leningr. uni-ta 19 (1973), 28-34 (in Russian).

[5] J. A. Hummel, Inequalities of Grunsky type for Aharonov pairs, J. Analyse Math. 25 (1972), 217-257.

[6] -, A variational method for Gel'fer functions, ibidem 30 (1976), 271-280.

[7] -, and M. Schiffer, Variational methods for Bieberbach-Eilenberg functions and for pairs, Ann. Acad. Sci. Fenn. AI, 3 (1977), 3-42.

[8] M. A. Lavrent'ev, $K$ teorii konformnych otobraženij, Trudy Matem. in-ta AN SSSR 5 (1934), 195-246 (in Russian).

[9] N. A. Lebedev, Princip plošadej v teorii odnolistnych funkcij, Nauka, Moskva 1975 (in Russian).

[10] Z. Lewandowski, R. Libera and E. Zlotkiewicz, Values assumed of Gel'fer functions, Ann. Univ. Mariae Curie-Skłodowska 31(1977), 75-84.

[11] M. Schiffer and D. C. Spencer, Functionals of finite Riemann surface, Princeton, New Jersey, 1954.

[12] A. Seiler, Inégalités de Grunsky du type Garabedian-Schiffer pour des paires de fonctions univalentes, C. R. Acad. Sci. Paris 283 (1976), 755-757.

[13] $O . T \mathrm{ammi}$, Grunsky type of inequalities and determination of the totality of the extremal functions, Ann. Acad. Sci. Fenn. AI, 443 (1969), 1-20.

[14] -, On extremum problems for bounded univalent functions, Reports of the Department of Mathematics University of Helsinki, ser. A. No. 13, 1977.

[15] K. Wlodarczyk, Inequalities of Grunsky-Nehari type for pairs of vector functions, Ann. Polon. Math. 37 (1980), 179-198.

[16] -, Coefficient inequalities and maximalization of some functionals for pairs of vector functions, ibidem 41 (1983), 1-26. 